\title{
The Therapeutic Effects of Mild to Moderate Intensity Aerobic Exercise on Glycemic Control in Patients with Type 2 Diabetes Mellitus: A Meta-Analysis of Randomized Trials
}

Siyao Gao $\cdot$ Jialing Tang $\cdot$ Guozhong Yi $\cdot$ Zhong Li $\cdot$ Zhenyin Chen $\cdot$

Ling Yu $\cdot$ Feng Zheng $\cdot$ Yajing Hu $\cdot$ Zhangui Tang

Received: August 5, 2021 / Accepted: August 25, 2021 / Published online: September 12, 2021

(C) The Author(s) 2021

\section{ABSTRACT}

Introduction: It has been recommended that physical activity be a part of treatment and management regimens of type 2 diabetes mellitus (T2DM), and research has shown that regular physical exercise facilitates glycemic control in these patients. In this analysis, our aim was to systematically show the therapeutic effects of mild to moderate intensity aerobic exercise on glycemic control in patients with T2DM.

Methods: From February to April 2021, we searched the https://www.clinicaltrials.gov, EMBASE, MEDLINE, Cochrane Central Register of Controlled Trials (CENTRAL), Web of Science

S. Gao · J. Tang $(\bowtie) \cdot$ G. Yi · Z. Li · Z. Chen $\cdot$ L. Yu F. Zheng . Y. Hu

Department of Physical Education, Central South University, Changsha 410083, Hunan, People's

Republic of China

e-mail: siyaog@163.com

S. Gao

e-mail: gaosiyao@csu.edu.cn

G. Yi

e-mail: 26987798@qq.com

Z. Li

e-mail: lizhong664@163.com

Z. Chen

e-mail: 472473468@qq.com and Google Scholar databases for trials that showed the effects of aerobic exercise on glycemic control in patients with T2DM. Glycated hemoglobin (HbA1c) was the endpoint in the analysis. The RevMan version 5.4 statistical program was used for statistical analysis, and the mean difference (MD) and 95\% confidence intervals (CI) used to represent the data following analysis.

Results: Eighteen trials involving 972 participants with T2DM were included in this metaanalysis, of whom 523 were assigned to an exercise group and 449 were assigned to a control group. A comparison pre- versus post-aerobic exercise showed that aerobic exercise significantly improved glycemic control (HbA1c) (MD 0.35, 95\% CI 0.23-0.48;

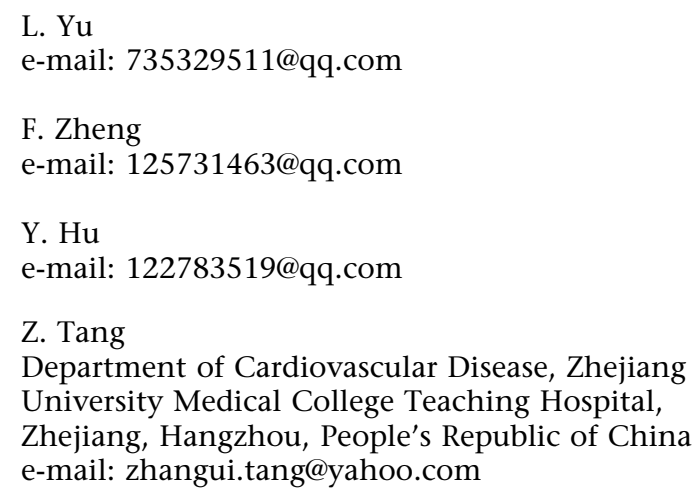


$P=0.00001)$ in these patients with T2DM. A second comparison, T2DM participants in the experimental group post-exercise versus T2DM participants from the control group at the end of the follow-up, also showed that aerobic exercise significantly improved glycemic control (MD - 0.46, 95\% CI -0.69 to -0.22 ; $P=0.0001)$. However, a comparison of HbA1c of T2DM participants in the control group at the beginning of the study compared to those at the end of follow-up did not show any significant improvement in glycemic control (MD $0.08,95 \%$ CI -0.05 to $0.21 ; P=0.21$ )

Conclusion: The current analysis showed that mild to moderate intensity aerobic exercise significantly improved glycemic control in patients with T2DM. Patients with T2DM who regularly participated in aerobic exercise activities had a better control of their disease than those who were not on a regular aerobic exercise regimen. These results lead to the recommendation that at least mild to moderate intensity aerobic exercise should be included in the treatment and management regimens of patients with T2DM.

Keywords: Type 2 diabetes mellitus; Aerobic exercise; Glycemic control; Glycosylated hemoglobin

\section{Key Summary Points}

\section{Why carry out this study?}

Physical activity has been recommended in the treatment and management of type 2 diabetes mellitus (T2DM), and research has shown glycemic control can be achieved with regular physical exercise.

The aim of this analysis was to systematically demonstrate the therapeutic effects of mild to moderate aerobic exercise on glycemic control in patients with T2DM.

\section{What was learned from the study?}

Mild to moderate aerobic exercise significantly improved glycemic control in patients with T2DM.

Patients with T2DM who performed aerobic exercise regularly achieved better disease control than those who did not.

These results lead to the recommendation that mild to moderate exercise should be included in the treatment and management regimens of patients with T2DM

\section{INTRODUCTION}

The prevalence of type 2 diabetes mellitus (T2DM) is on the rise [1], and given the unhealthy kind of lifestyle that has been adopted by many people around the globe [2], a decline in the number of patients with T2DM is not expected. Globally, 425 million people are living with T2DM, and a further rise is inevitable in the coming years. Over the past three decades, the total number of people with T2DM has more than doubled worldwide, with the results that T2DM is now considered to be one of the most important challenges faced by the public health systems of all nations [3]. A recently developed population-level T2DM 
mathematical model, fitted to six populationbased survey data collected between 1990 and 2017, was used to characterize T2DM in Jordan [4]. This model showed that T2DM prevalence in Jordan was $14.0 \%$ in 1990, reached approximately $16.0 \%$ in 2020 and would reach $20.6 \%$ in 2050. The total predicted number of new cases of T2DM in Jordan was 12,313 in 1990, 36,941 in 2020 and 79,419 by 2050 [4]. The authors of another study recommended that lifestyle modifications, including weight loss, physical exercise, healthy diet and smoking cessation, should be implemented to prevent the risk of cardiovascular and other major complications in such patients [5]. Physical activity has been recommended in the treatment and management of T2DM [6], and research has shown that glycemic control can be achieved with regular physical exercise. The aim of analysis presented here was to systematically show the therapeutic effects of mild to moderate intensity aerobic exercise on glycemic control in patients with T2DM.

\section{METHODS}

\section{Databases and Search Strategies}

Between February and April 2021, we searched the https://www.clinicaltrials.gov, EMBASE, MEDLINE, Cochrane Central Register of Controlled Trials (CENTRAL), Web of Science and Google Scholar databases for trials showing the effects of aerobic exercise on glycemic control in patients with T2DM. The following keywords and phrases were used to search the databases for relevant articles: "diabetes mellitus and exercise," "diabetes mellitus and aerobic exercise," "diabetes mellitus and glycemic control and exercise," "diabetes mellitus and exercise intervention," "diabetes mellitus and physical exercise," "diabetes mellitus and exercise and HbA1c (glycated hemoglobin)," "physical exercise and HbA1c," "physical activity and diabetes mellitus," "physical activity and HbA1c".

\section{Inclusion and Exclusion Criteria}

Studies were included if: (1) they were randomized trials showing the effect of aerobic exercise on glycemic control in patients with T2DM; (2) they involved an experimental (aerobic exercise) group and a control (non-exercise) group; and (3) HbA1c was reported as the endpoint.

Studies were excluded if: (1) they were nonrandomized studies (i.e. observational studies, systematic reviews, meta-analyses, literature reviews and case studies, among others); (2) they included only high-intensity aerobic exercise; and (3) HbA1c was not reported.

\section{Data Extraction and Quality Assessment}

Eight reviewers independently extracted data from all trials that met the inclusion criteria. The extracted data included: first author's name; year of publication; time period of participants' enrollment; total number of participants with T2DM who were assigned to the aerobic exercise and the control groups, respectively; HbA1c at baseline, pre-exercise and post-exercise; the type of aerobic exercise intervention; the duration of the follow-up; the type of study; and the methodological features of the trials. Any disagreement regarding the data was discussed with the corresponding author who made the final decision.

Since all studies included in this analysis were randomized trials, the methodological quality was assessed following the recommendations of the Cochrane Collaboration [7], with a grade given to each trial as follows: grade ' $A$ ' indicated a low risk of bias; grade ' $\mathrm{B}$ ' indicated a moderate risk of bias; and grade ' $\mathrm{C}$ ' indicated a high risk of bias.

\section{Statistical Analysis}

The statistical analysis was carried out using the RevMan software version 5.4. Since continuous data, including means and standard deviations, were reported in the original studies, the mean difference (MD) with the respective 95\% confidence interval (CI) was used to represent the 
results following analysis. Heterogeneity was assessed by the $Q$ statistic test whereby a $P$ value $\leq 0.05$ was considered to be statistically significant. Any $P$ value $>0.05$ was considered to indicate non-insignificance. Heterogeneity was also assessed by the $I^{2}$ statistic test, whereby a lower $I^{2}$ value denoted a lower heterogeneity. A fixed statistical effect model was used if $I^{2}<$ $50 \%$, and a random statistical effect model was used if $I^{2}>50 \%$.

A sensitivity analysis was also carried out by an exclusion method, and each trial was excluded one by one and a new analysis was carried out each time to observe for any significant change in result.

\section{Compliance with Ethical Guidelines}

This article is based on previously conducted studies and does not contain any new studies with human or animal participants performed by any of the authors.

\section{RESULTS}

\section{Search Outcomes}

The Preferred Reporting Items in Systematic Reviews and Meta-Analyses (PRISMA) reporting guideline was followed [8]. Our search of the above-mentioned databases resulted in the identification of 24,836 publications. After a careful assessment of the titles and abstracts, 24,410 publications were excluded since they were irrelevant to the topic under evaluation, leaving 426 full-text articles to be assessed for eligibility. Ultimately, only 18 trials [9-26] were selected for inclusion in this analysis, with the other 408 studies excluded for the following reasons:

- Non-randomized studies $(n=26)$;

- Systematic reviews, meta-analyses, literature reviews, case studies $(n=68)$;

- Did not report the endpoint (HbA1c) which was under consideration $(n=14)$;

- A control group was absent $(n=21)$;

- Non-aerobic exercises or very intense training $(n=48)$;
- Duplicated studies $(n=231)$.

The flow diagram of study inclusion is shown in Fig. 1.

\section{Endpoints, Follow-Up and Type of Interventions}

The endpoints, duration of the follow-up and the type of aerobic exercise intervention performed by the participants are shown in Table 1. Based on the data in Table 1, we considered a mean follow-up time period ranging from 8 to 104 weeks. HbA1c (\%) was considered to be the endpoint.

HBA1c was assessed in patients with T2DM as: HbA1c in those assigned to the aerobic exercise group (pre-exercise vs. post-exercise); HbA1c in the post-exercise group versus the control group at the end of the study; and HbA1c in the control group at the start/baseline versus $\mathrm{HbA1c}$ at the end of the follow-up in the control group.

\section{General and Baseline Features of the Trials}

The general features of the trials are listed in Table 1. Eighteen trials involving 972 participants with T2DM were included in this metaanalysis, of whom 523 were assigned to an exercise group and 449 were assigned to a control group. The time period of patients' enrollment ranged from 1999 to 2018.

The baseline features of the participants are listed in Table 2 . The mean age of the participants ranged from 47.2 to 70.2 years. Four studies reported only female patients, gender was not mentioned in four studies and in the remaining ten studies, the mean percentage of male participants varied from 31.8 to $60.0 \%$ (Table 2 . The mean body mass index also varied from 25.0 to $36.7 \mathrm{~kg} / \mathrm{m}^{2}$. Mean HbA1c at baseline varied from 46.0 to $79.0 \mathrm{mmol} / \mathrm{mol}$.

\section{Main Result of this Analysis}

Comparison of groups pre- versus post-aerobic exercise showed that mild to moderate intensity aerobic exercise significantly improved 


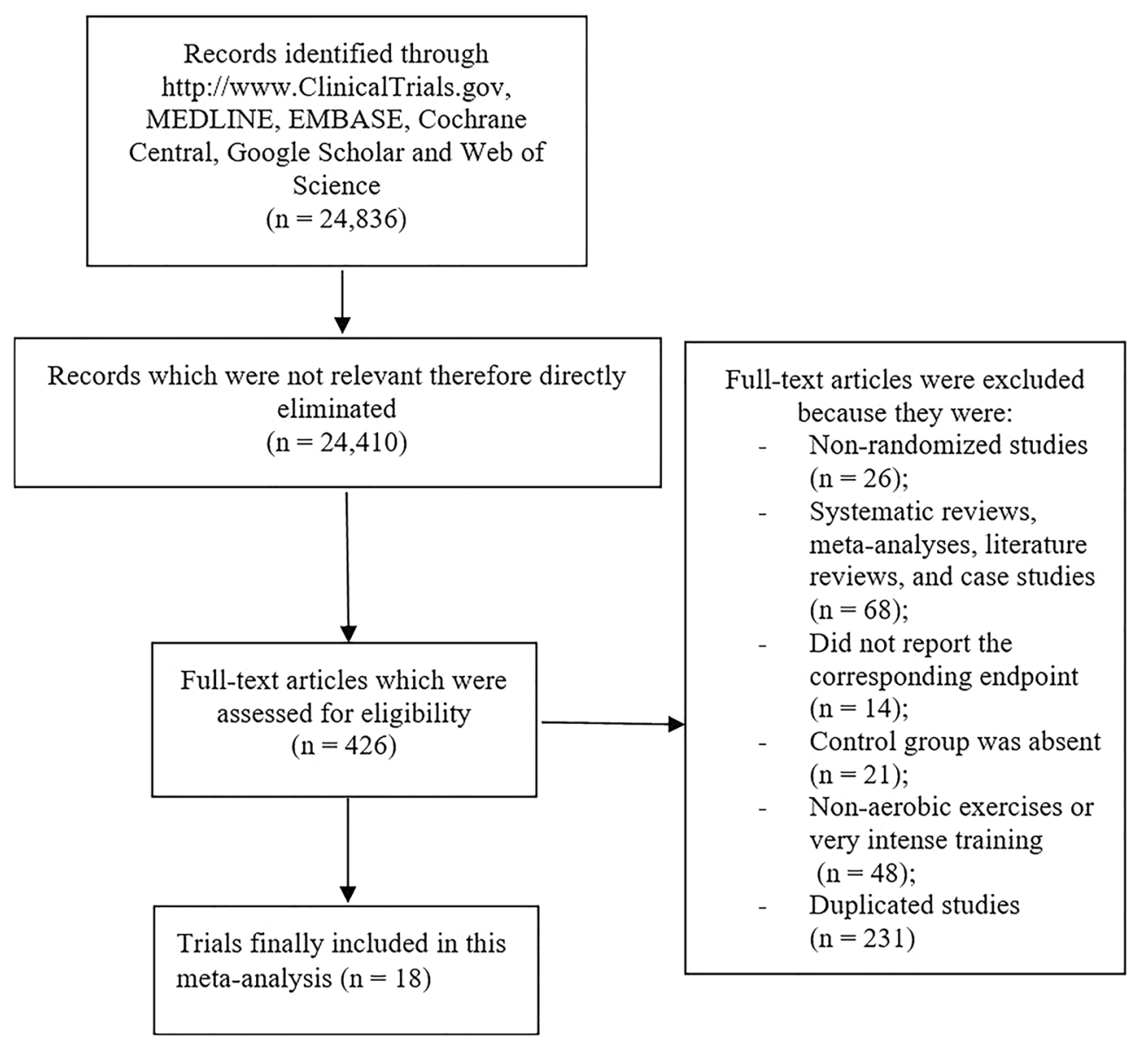

Fig. 1 Study selection flow diagram

glycemic control (HbA1c) (MD 0.35, 95\% CI $0.23-0.48 ; P=0.00001$ ) in these patients with T2DM, as shown in Fig. 2a, b.

A second comparison was betweenh T2DM participants post exercise (HbA1c at the end of the study) versus the control group (HbA1c at the end of follow-up). This analysis showed that aerobic exercise significantly improved glycemic control (MD $-0.46,95 \% \mathrm{CI}-0.69$ to $-0.22 ; P=0.0001])$ in these patients with T2DM, as shown in Fig. 3a, b.

However, when a comparison of HbA1c was carried out in participants within the control group at the beginning of the study vs. at the end of the follow-up (only on participants who were not assigned to aerobic exercise), our analysis did not show any significant improvement in glycemic control (MD 0.08, 95\% CI -0.05 to $0.21 ; P=0.21$ ), as shown in Fig. $4 a$, b.
The study of Loreto et al. [19] had a followup period of 104 weeks; all other studies included in this analysis had a shorter follow-up time period. Therefore, we carried out an analysis that excluded this study and found that there were no significant changes in the results when compared to the results obtained with all studies included. We then performed an analysis excluding the study of Loreto et al. [19] in which we compared pre- and post-aerobic exercise; the results still showed that mild to moderate intensity aerobic exercise significantly improved glycemic control (MD 0.48, 95\% CI $0.28-0.67 ; P=0.00001)$. When T2DM participants were compared post-exercise versus the control group, the results remained the same (MD - 0.52, 95\% CI -0.76 to -0.27 ; $P=0.0001)$. Finally, a comparison of the control group at the beginning of the intervention versus the end of the follow-up also did not 


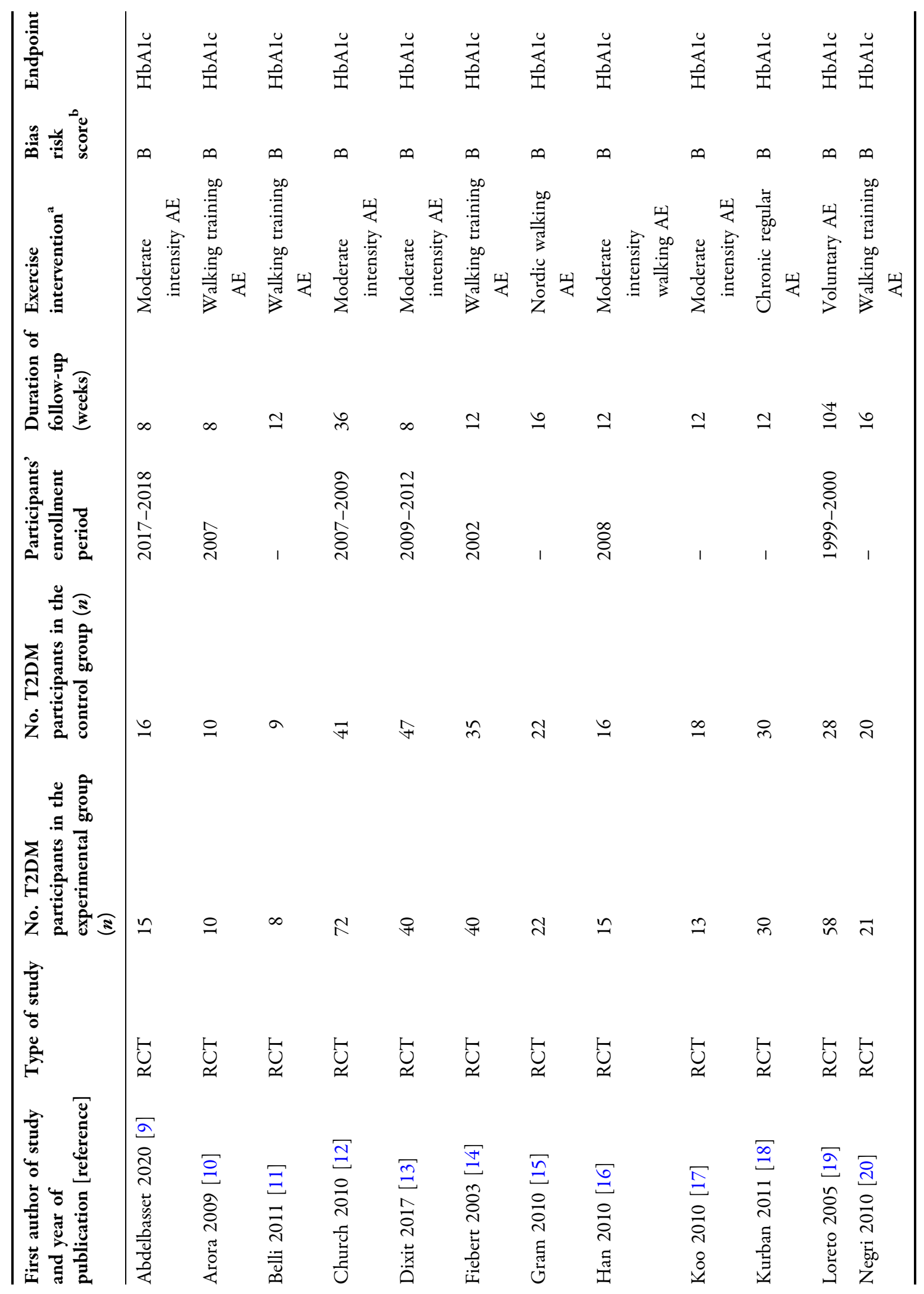




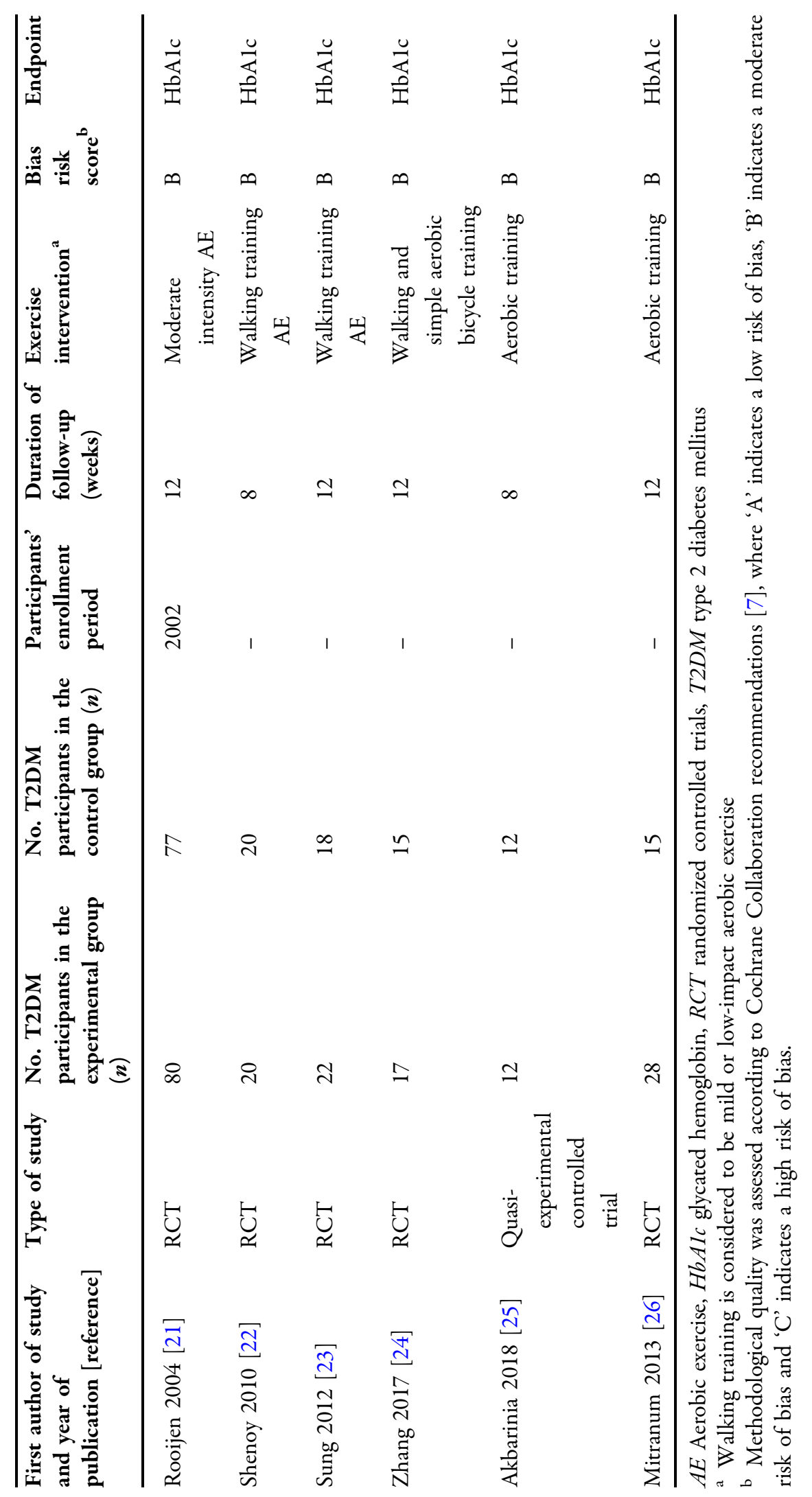




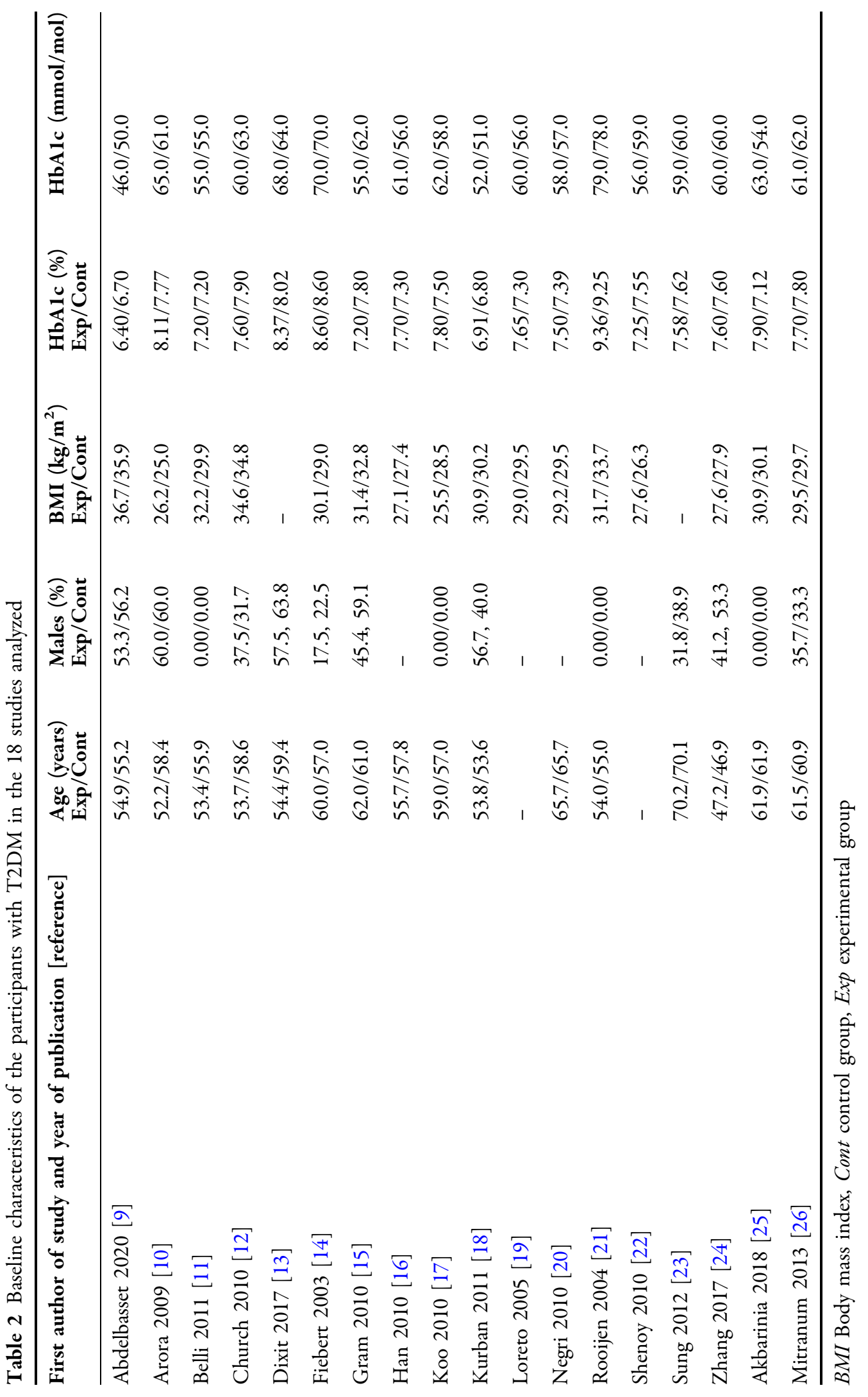




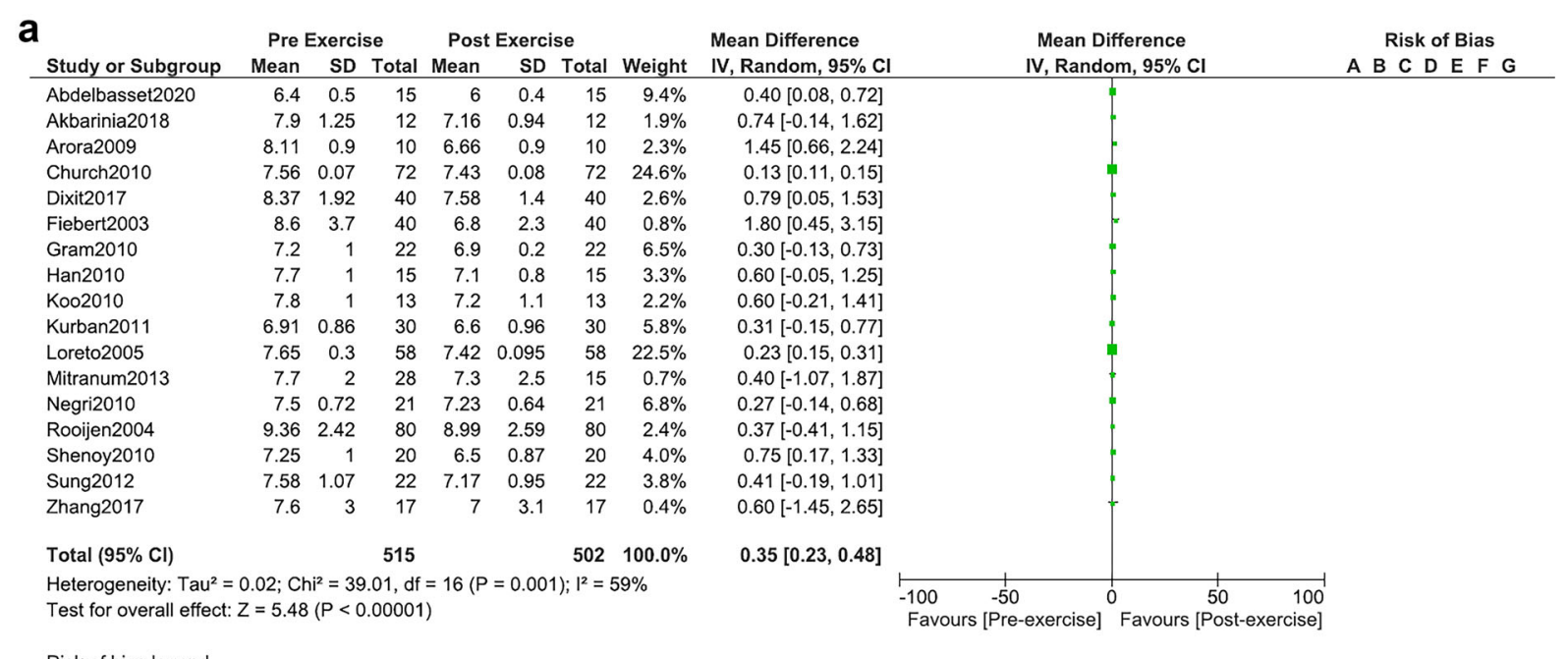

Risk of bias legend

(A) Random sequence generation (selection bias)

(B) Allocation concealment (selection bias)

(C) Blinding of participants and personnel (performance bias)

(D) Blinding of outcome assessment (detection bias)

(E) Incomplete outcome data (attrition bias)

(F) Selective reporting (reporting bias)

(G) Other bias

b

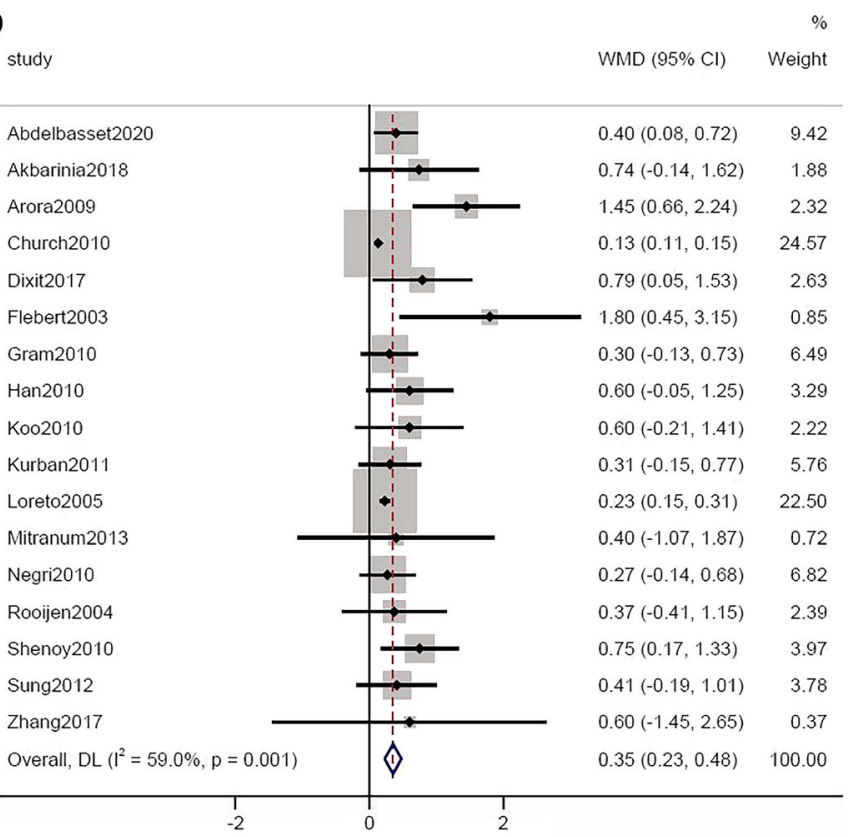

NOTE: Weights are from random-effects model

Fig. 2 a Glycemic control (HbAlc) in patients preexercise versus post-exercise intervention. $\mathbf{b} \mathrm{HbAlc}$ preexercise versus post-exercise intervention (Stata statistical

show any significant improvement in HbA1c (MD 0.11, 95\% CI -0.07 to $0.28 ; P=0.23$ ).

A sensitivity analysis was carried out using the RevMan software program in which each software; StataCorp, TX, USA). CI Confidence interval, $S D$ standard deviation, WMD Weighted mean difference

trial was excluded one by one, in turn, in the data input section and a new analysis was carried out each time to be compared with the final result. Again, consistent results were obtained 
a

Aerobic Exercise Control Mean Difference

\begin{tabular}{lccccccccc} 
Study or Subgroup & Mean & SD & Total & Mean & SD & Total & Weight & IV, Random, $95 \%$ CI \\
\hline 1.1.1 Experiment versus control group & & & & & & \\
Abdelbasset2020 & 6 & 0.4 & 15 & 6.5 & 0.5 & 16 & $7.9 \%$ & $-0.50[-0.82,-0.18]$
\end{tabular}

Mean Difference

Risk of Bias

Abdelbasset2020

Akbarinia2018

$\begin{array}{rrrrrrr}7.16 & 0.94 & 12 & 7.34 & 1.38 & 12 & 3.6 \% \\ 6.66 & 0.9 & 10 & 7.41 & 0.9 & 10 & 4.5 \%\end{array}$

$\begin{array}{lrrrrrrr}\text { Arora2009 } & 6.66 & 0.9 & 10 & 7.41 & 0.9 & 10 & 4.5 \% \\ \text { Belli2011 } & 5.9 & 0.2 & 8 & 7.2 & 0.7 & 9 & 6.6 \%\end{array}$

Church2010

Dixit2017

$\begin{array}{ll}7.43 & 0.08\end{array}$

Fiebert2003

$\begin{array}{rrrrrrr}7.58 & 1.4 & 40 & 8.03 & 1.46 & 47 & 5.7 \%\end{array}$

$-0.18[-1.12,0.76]$

$-0.75[-1.54,0.04]$

$-1.30[-1.78,-0.82]$

$-0.31[-0.35,-0.27]$

$\begin{array}{rrrrrrr}6.8 & 2.3 & 40 & 8.2 & 2.3 & 35 & 3.2 \%\end{array}$

Gram2010

Han2010

Koo2010

$\begin{array}{lllll}6.9 & 0.2 & 22 & 7.9 & 0.3\end{array}$

$-0.45[-1.05,0.15]$

$-1.40[-2.44,-0.36]$

$-1.00[-1.15,-0.85]$

$-0.10[-0.70,0.50]$

Kurban2011

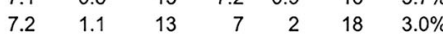

$0.20[-0.90,1.30]$

$-0.29[-0.82,0.24]$

Loreto2005

Mitranum2013

Negri2010

$\begin{array}{lllll}6.6 & 0.96 & 30 & 6.89 & 1.11\end{array}$

$\begin{array}{ll}7.42 & 0.345\end{array}$

Rooijen2004

Shenoy 2010

Sung2012

Zhang 2017

$\begin{array}{rr}7.3 & 2.5 \\ 7.23 & 0.64\end{array}$

$\begin{array}{ll}7.23 & 0.64 \\ 8.99 & 2.59\end{array}$

$\begin{array}{ll}6.5 & 0.87\end{array}$

$\begin{array}{ll}7.17 & 0.95\end{array}$

$\begin{array}{rrr}58 & 7.27 & 0.21 \\ 28 & 8.1 & 2\end{array}$

$\begin{array}{lll}21 & 7.34 & 0.53\end{array}$

$\begin{array}{lll}80 & 8.26 & 1.97\end{array}$

$\begin{array}{rrr}80 & 8.26 & 1.97 \\ 20 & 7.5 & 0.9\end{array}$

$\begin{array}{lll}22 & 8.12 & 1.41\end{array}$

Subtotal $(95 \% \mathrm{Cl})$

$\begin{array}{lllll}17 & 7.3 & 2.9 & 15 & 18 \%\end{array}$

$0.15[0.03,0.27]$

$-0.80[-2.17,0.57]$

$-0.11[-0.47,0.25]$

$0.73[0.01,1.45]$

$-1.00[-1.55,-0.45]$

$-0.95[-1.71,-0.19]$

$-0.30[-2.38,1.78]$

$-0.46[-0.69,-0.22]$

Heterogeneity: $\mathrm{Tau}^{2}=0.15 ; \mathrm{Chi}^{2}=182.18, \mathrm{df}=17(\mathrm{P}<0.00001) ; \mathrm{I}^{2}=91 \%$

Test for overall effect: $Z=3.85(P=0.0001)$

Total $(95 \% \mathrm{Cl})$

523

$449 \quad 100.0 \% \quad-0.46[-0.69,-0.22]$

Heterogeneity: Tau $^{2}=0.15 ; \mathrm{Chi}^{2}=182.18, \mathrm{df}=17(\mathrm{P}<0.00001) ; \mathrm{I}^{2}=91 \%$

Test for overall effect: $Z=3.85(P=0.0001)$

Test for subgroup differences: Not applicable

IV, Random, $95 \% \mathrm{Cl}$

A B C D E F G

Risk of bias legend

(A) Random sequence generation (selection bias)

(B) Allocation concealment (selection bias)

(C) Blinding of participants and personnel (performance bias)

(D) Blinding of outcome assessment (detection bias)

(E) Incomplete outcome data (attrition bias)

(F) Selective reporting (reporting bias)

(G) Other bias

b

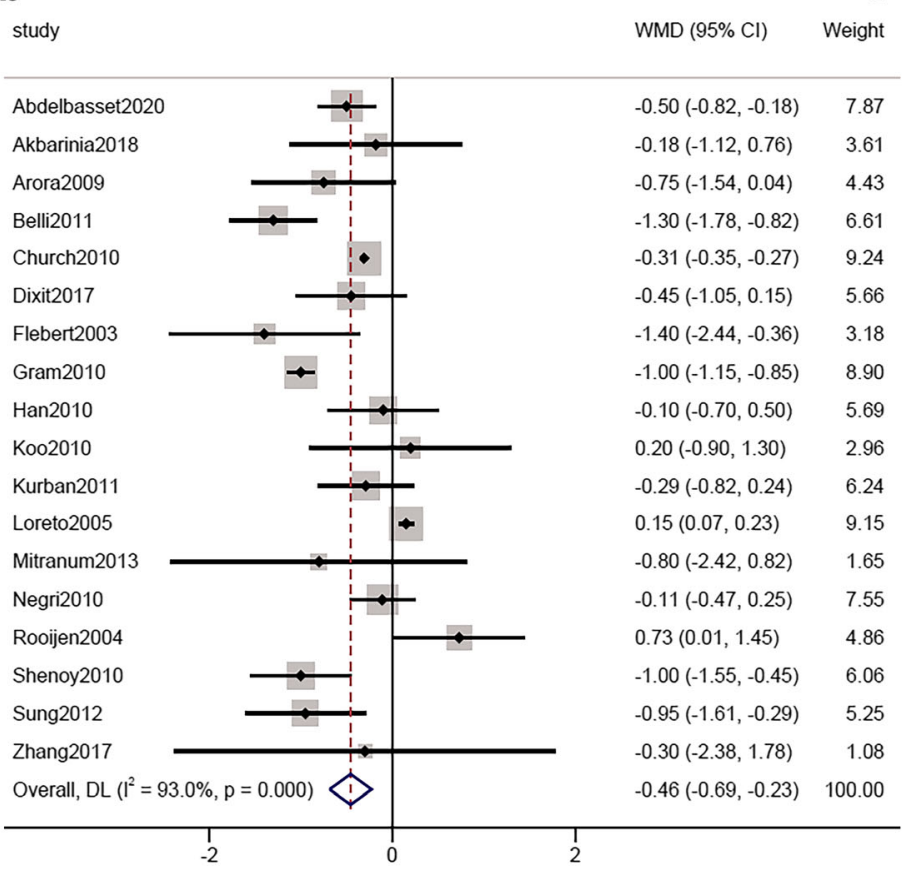

NOTE: Weights are from random-effects model

Fig. 3 a HbAlc in the exercise intervention vs. control group. $\mathbf{b} \mathrm{HbAlc}$ in the exercise intervention vs. the control group (STATA) 


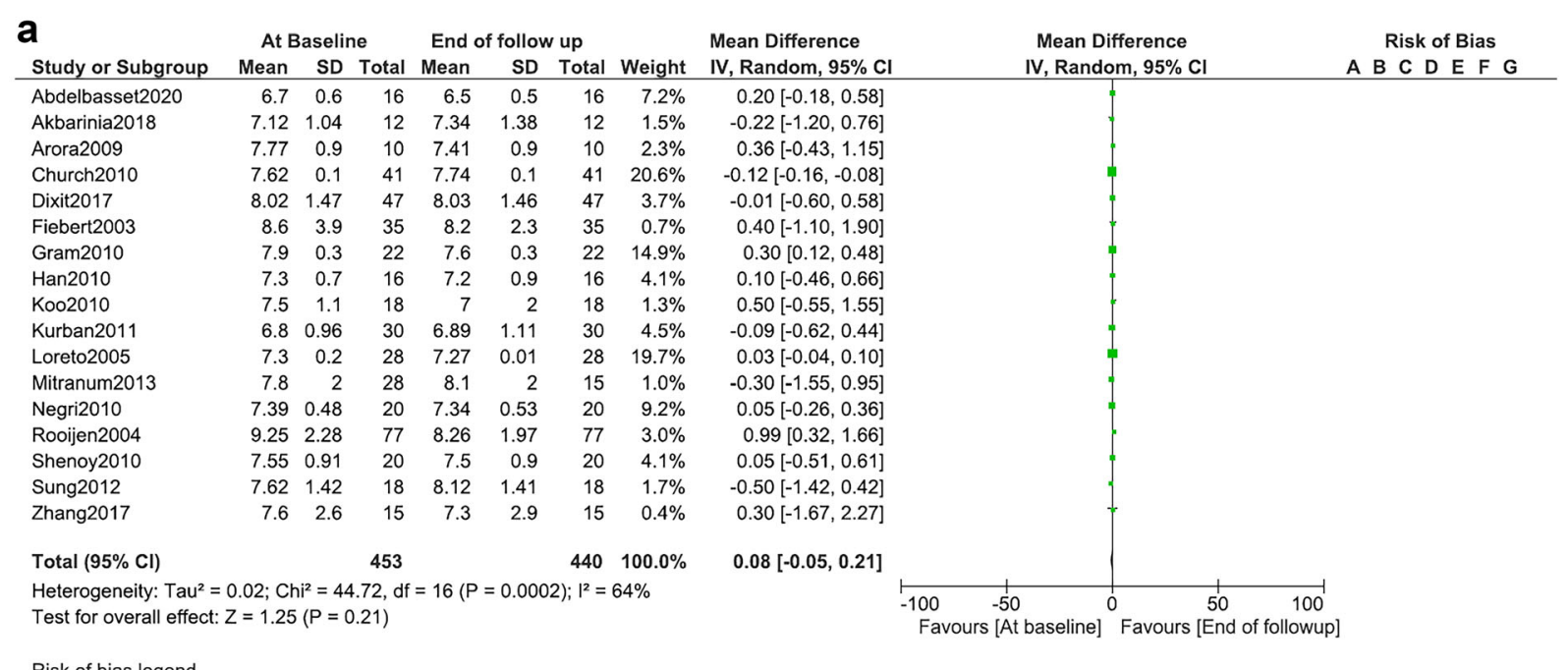

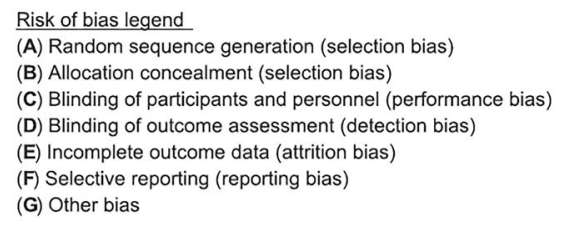

\section{b}

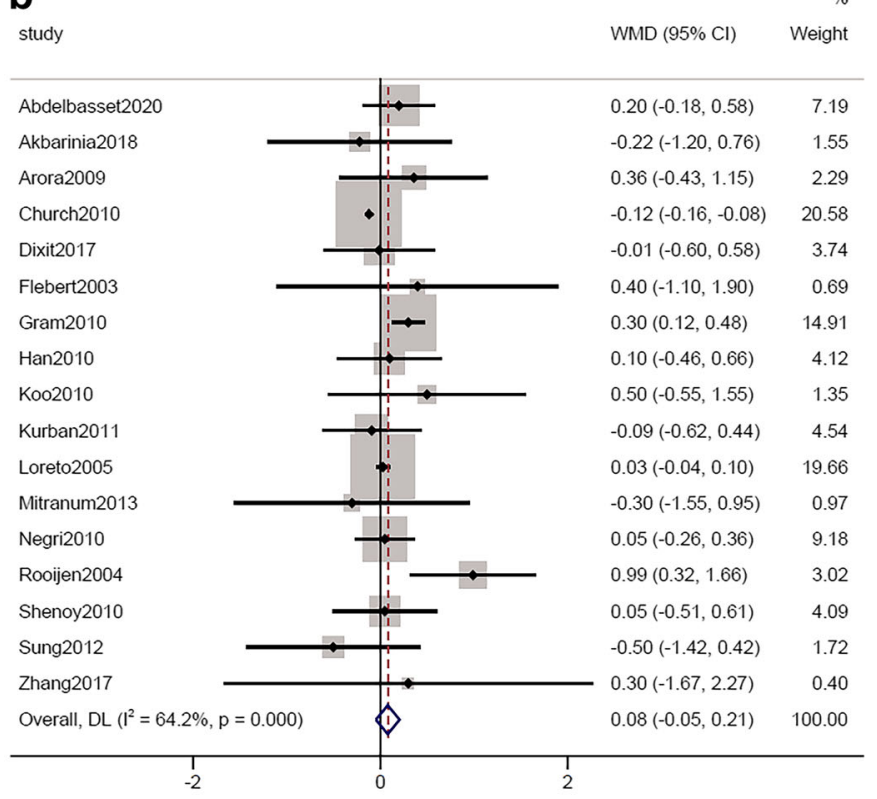

NOTE: Weights are from random-effects model

Fig. 4 a HbAlc in the control group at baseline versus at the end of the study intervention. $\mathbf{b}$ HbAlc in the control group at baseline versus at the end of the study intervention (STATA)

throughout. The results for the sensitivity analysis were not significantly different from the main results.

Publication bias is shown in Fig. 5.

\section{DISCUSSION}

Our results demonstrate that a significantly improved glycemic control was achieved by patients with T2DM who were assigned to a 


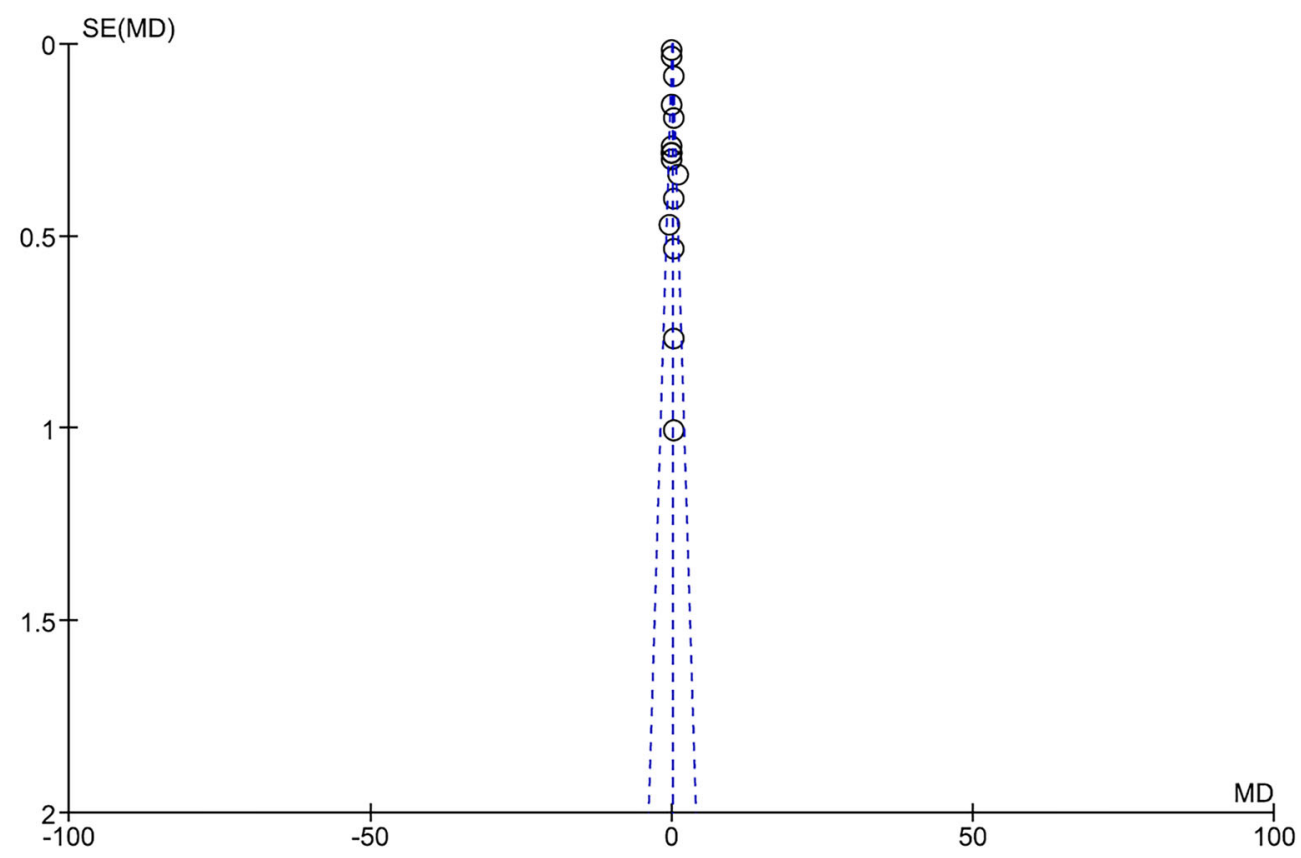

Fig. 5 Funnel plot showing publication bias

mild to moderate intensity aerobic exercise group in comparison to the control group. Different categories of comparisons were made: pre- versus post-exercise in the interventional group; the exercise group versus the control group at the end of the follow-up period; and participants in the control group at the beginning (baseline) versus at the end of the followup time period.

The results of our analysis involved a high level of heterogeneity due to several confounding factors. Information bias, which results in systematic differences in the manner data on exposure or outcomes are obtained from the different study groups, associated with incorrect estimations and errors or small variations in the measurement of HbA1c, and the different kinds of aerobic exercises with different intensities in each specific study, considered to be misclassification, may have had an influence on the final results. Selection bias, consisting of sampling bias in the original studies and during data extraction for the present current meta-analysis, might also have contributed to the high heterogeneity in our current analysis. Interaction of other factors, such as comorbidities (e.g. obesity, smoking status, dyslipidemia) might also have contributed to this high level of heterogeneity.

In support of the results of this analysis, in a study performed by Shakil-Ur-Rehman et al., a supervised structured aerobic exercise training program in patients with T2DM showed that aerobic exercise was significantly effective in improving blood sugar control compared to a non-exercise control group, with this improvement seen in both male and female participants [27]. Furthermore, a secondary analysis of a randomized controlled trial demonstrating physical activity and glycemic control in Chinese patients with T2DM also supported the results of this current analysis, with a significant improvement in HbA1c achieved by those patients who were assigned to an exercise intervention group [28].

In another recent systematic review and meta-analysis by Shah et al. [29], which aimed to show the therapeutic effects of exercise and general physical activity on glycemic control in patients with T2DM, the authors reported that exercise played a major role in optimizing glycemic control and improving quality of life, waist circumference and body mass index in their patients with T2DM, leading them to 
recommend physical activity in the daily life of such patients. Their meta-analysis was focused on general physical activity, including aerobic as well as intense anaerobic exercises, and was also based on the assessment of the effect of exercise on other parameters [29]. Our current analysis is differs from that of Shah et al. [29] in that it is strictly limited to the effects of mild to moderate intensity aerobic exercise and its specific impact on glycemic control only. Many patients with T2DM have other co-morbidities, such as obesity and health complications, including cardiovascular diseases, which do not allow them to carry out intense exercises or which restrict their ability to carry out intense physical activities. Therefore, we believe that mild to moderate intensity aerobic exercise might be more appropriate in these patients. A systematic review and the joint AMD/SID/ SISMES evidence-based practical guideline based on walking for subjects with T2DM [30] recommend at least low-impact aerobic exercise. and the authors stated that there is sufficient evidence to show walking or low-impact aerobic exercise to be beneficial.

Research has shown that walking is the major activity of choice in patients with T2DM [29]. While our analysis showed mild to moderate intensity aerobic exercise had a positive effect on glycemic control, such exercises could also promote weight loss and maintenance. Moderate intensity physical activity at least 30 min per day should be effective and could result in favorable long-term cardiovascular adaptations in patients with T2DM.

Apart from glycemic control, in another study [31, 32] examining the effects of aerobic exercise on abdominal fat, thigh muscle mass and muscle strength in diabetic subjects, moderate intensity aerobic exercise on a daily basis was found to be effective in reducing abdominal fat mass and other benefits. These results indicate that aerobic exercise in patients with T2DM result in a better control of their overall health status. In addition, aerobic training could improve platelet functions in patients with T2DM, thereby preventing platelet hyperreactivity [25].

\section{Limitations}

This study has a number of limitations. First, the total number of patients with T2DM were limited in comparison to the number included in other meta-analyses, which could have had an impact on the final outcome. Second, the follow-up time period was not similar in all studies, which may also have affected the results of this analysis. Third, any oral hypoglycemic agents or insulin used for the control of blood sugar, as well as for diet control, were not taken into account in the present analysis; co-morbidities were also ignored. Fourth, apart from walking, few studies included other forms of exercise, such as cycling; however, all of these different types of aerobic exercises could not be separately assessed because the number of studies reporting certain exercises were limited and there would not be sufficient numbers of patients for comparison, thus leading to inconclusive outcomes. Furthermore, a moderate risk of heterogeneity was observed, which could have been due to several confounding factors.

\section{CONCLUSION}

The present analysis showed that mild to moderate intensity aerobic exercise significantly improved glycemic control in patients with T2DM. Patients with T2DM who participated in regular aerobic exercise programs had a better control of their disease than those who were not on a regular aerobic exercise regimen. These results lead to the recommendation that at least mild to moderate intensity aerobic exercise should be included in the treatment and management of patients with T2DM.

\section{ACKNOWLEDGEMENTS}

Funding. No external funding or sponsorship was received for this study or publication of this article. This study was funded by the authors. 
Authorship. All named authors meet the International Committee of Medical Journal Editors (ICMJE) criteria for authorship for this article, take responsibility for the integrity of the work as a whole, and have given their approval for this version to be published. No medical writing or editorial assistance was required.

Authors' Contributions. SG, JT, GY, ZL, ZC, $\mathrm{LY}, \mathrm{FZ}, \mathrm{YH}$ and ZT were responsible for the conception and design of the study, acquisition, analysis and interpretation of the data, drafting of the initial manuscript and critical revision for important intellectual content. All authors approved the final manuscript as it is.

Disclosures. Siyao Gao, Jialing Tang, Guozhong $\mathrm{Yi}$, Zhong Li, Zhenyin Chen, Ling $\mathrm{Yu}$, Feng Zheng, Yajing $\mathrm{Hu}$ and Zhangui Tang declare that they have no competing interests.

Compliance with Ethical Guidelines. This meta-analysis is based on previously conducted studies and does not contain any new study with human participants or animals performed by any of the authors.

Data Availability. All data generated or analyzed during this study are included in this published article. References of the original papers involving the data source which have been used in this paper have been listed in the main text of this current manuscript. All data are publicly available in electronic databases.

Open Access. This article is licensed under a Creative Commons Attribution-NonCommercial 4.0 International License, which permits any non-commercial use, sharing, adaptation, distribution and reproduction in any medium or format, as long as you give appropriate credit to the original author(s) and the source, provide a link to the Creative Commons licence, and indicate if changes were made. The images or other third party material in this article are included in the article's Creative Commons licence, unless indicated otherwise in a credit line to the material. If material is not included in the article's Creative Commons licence and your intended use is not permitted by statutory regulation or exceeds the permitted use, you will need to obtain permission directly from the copyright holder. To view a copy of this licence, visit http://creativecommons.org/licenses/by$\mathrm{nc} / 4.0 /$.

\section{REFERENCES}

1. Lovic D, Piperidou A, Zografou I, et al. The growing epidemic of diabetes mellitus. Curr Vasc Pharmacol. 2020;18(2):104-9.

2. Moon S. Unhealthy lifestyle behaviors in Korean People with metabolic syndrome. J Community Health Nurs. 2017;34(2):69-79.

3. Chen L, Magliano DJ, Zimmet PZ. The worldwide epidemiology of type 2 diabetes mellitus-present and future perspectives. Nat Rev Endocrinol. 2011;8(4):228-36.

4. Awad SF, Huangfu P, Dargham SR, et al. Characterizing the type 2 diabetes mellitus epidemic in Jordan up to 2050. Sci Rep. 2020;10(1):21001.

5. Afroz A, Ali L, Karim MN, et al. Glycaemic control for people with type 2 diabetes mellitus in Bangladesh-an urgent need for optimization of management plan. Sci Rep. 2019;9(1):10248.

6. Lumb A. Diabetes and exercise. Clin Med (Lond). 2014;14(6):673-6.

7. Higgins JPT, Altman DG, Gøtzsche PC, et al. The Cochrane Collaboration's tool for assessing risk of bias in randomised trials. BMJ. 2011;343:d5928.

8. Liberati A, Altman DG, Tetzlaff J, et al. The PRISMA statement for reporting systematic reviews and meta-analyses of studies that evaluate healthcare interventions: explanation and elaboration. BMJ. 2009;339:b2700.

9. Abdelbasset WK, Tantawy SA, Kamel DM, et al. Effects of high-intensity interval and moderate-intensity continuous aerobic exercise on diabetic obese patients with nonalcoholic fatty liver disease: a comparative randomized controlled trial. Medicine (Baltimore). 2020;99(10):e19471.

10. Arora E, Shenoy S, Sandhu JS. Effects of resistance training on metabolic profile of adults with type 2 diabetes. Indian J Med Res. 2009;129(5):515-9.

11. Belli T, Ribeiro LFP, Ackermann MA, Baldissera V, Gobatto CA, da Silva RG. Effects of 12-week 
overground walking training at ventilatory threshold velocity in type 2 diabetic women. Diabetes Res Clin Pract. 2011;93(3):337-43.

12. Church TS, Blair SN, Cocreham S, et al. Effects of aerobic and resistance training on hemoglobin A1c levels in patients with type 2 diabetes: a randomized controlled trial. JAMA. 2010;304(20):2253-62.

13. Dixit S, Maiya A, Shastry BA. Effect of moderateintensity aerobic exercise on glycosylated haemoglobin among elderly patients with type 2 diabetes and peripheral neuropathy. Indian J Med Res. 2017;145(1):129-32.

14. Goldhaber-Fiebert JD, Goldhaber-Fiebert SN, Tristán ML, Nathan DM. Randomized controlled community-based nutrition and exercise intervention improves glycemia and cardiovascular risk factors in type 2 diabetic patients in rural Costa Rica. Diabetes Care. 2003;26(1):24-9.

15. Gram B, Christensen R, Christiansen C, Gram J. Effects of nordic walking and exercise in type 2 diabetes mellitus: a randomized controlled trial. Clin J Sport Med. 2010;20(5):355-61.

16. $\mathrm{Ku} \mathrm{YH,} \mathrm{Han} \mathrm{KA,} \mathrm{Ahn} \mathrm{H}$, et al. Resistance exercise did not alter intramuscular adipose tissue but reduced retinol-binding protein- 4 concentration in individuals with type 2 diabetes mellitus. J Int Med Res May-Jun. 2010;38(3):782-91.

17. Koo BK, Han KA, Ahn HJ, Jung JY, Kim HC, Min KW. The effects of total energy expenditure from all levels of physical activity vs. physical activity energy expenditure from moderate-to-vigorous activity on visceral fat and insulin sensitivity in obese type 2 diabetic women. Diabet Med. 2010;27(9):1088-92.

18. Kurban S, Mehmetoglu I, Yerlikaya HF, Gönen S, Erdem S. Effect of chronic regular exercise on serum ischemia-modified albumin levels and oxidative stress in type 2 diabetes mellitus. Endocr Res. 2011;36(3):116-23.

19. Di Loreto C, Fanelli C, Lucidi P, et al. Make your diabetic patients walk: long-term impact of different amounts of physical activity on type 2 diabetes. Diabetes Care. 2005;28(6):1295-302.

20. Negri C, Bacchi E, Morgante S, et al. Supervised walking groups to increase physical activity in type 2 diabetic patients. Diabetes Care. 2010;33(11):2333-5.

21. van Rooijen AJ, Rheeder P, Eales CJ, Becker PJ. Effect of exercise versus relaxation on haemoglobin A1C in Black females with type 2 diabetes mellitus. QJM. 2004;97(6):343-51.

22. Shenoy S, Guglani R, Sandhu JS. Effectiveness of an aerobic walking program using heart rate monitor and pedometer on the parameters of diabetes control in Asian Indians with type 2 diabetes. Prim Care Diabetes. 2010;4(1):41-5.

23. Sung K, Bae S. Effects of a regular walking exercise program on behavioral and biochemical aspects in elderly people with type II diabetes. Nurs Health Sci. $2012 ; 14(4): 438-45$.

24. Zhang L-Y, Liu T, Teng Y-Q, et al. Effect of a 12 -week aerobic exercise training on serum fetuin-a and adipocytokine levels in type 2 diabetes. Exp Clin Endocrinol Diabetes. 2018;126(8):487-92.

25. Akbarinia A, Kargarfard M, Naderi M. Aerobic training improves platelet function in type 2 diabetic patients: role of microRNA-130a and GPIIb. Acta Diabetol. 2018;55(9):893-9.

26. Mitranun W, Deerochanawong C, Tanaka H, Suksom D. Continuous vs interval training on glycemic control and macro- and microvascular reactivity in type 2 diabetic patients. Scand J Med Sci Sports. 2014;24(2):e69-76.

27. Shakil-Ur-Rehman S, Karimi H, Gillani SA, Amjad I, Ahmad S, Yaseen A. Response to a supervised structured aerobic exercise training program in patients with type 2 diabetes mellitus-does gender make a difference? A randomized controlled clinical trial. J Natl Med Assoc. 2018;110(5):431-9.

28. Yao W-Y, Han M-G, De Vito G, et al. Physical activity and glycemic control status in chinese patients with type 2 diabetes: a secondary analysis of a randomized controlled trial. Int J Environ Res Public Health. 2021;18(8):4292.

29. Shah SZA, Karam JA, Zeb A, et al. Movement is improvement: the therapeutic effects of exercise and general physical activity on glycemic control in patients with type 2 diabetes mellitus: a systematic review and meta-analysis of randomized controlled trials. Diabetes Ther. 2021;12(3):707-32.

30. Moghetti P, Balducci S, Guidetti L, et al. Walking for subjects with type 2 diabetes: a systematic review and joint AMD/SID/SISMES evidence-based practical guideline. Nutr Metab Cardiovasc Dis. 2020;30(11):1882-98.

31. Ford ES, Herman WH. Leisure-time physical activity patterns in the U.S. diabetic population. Findings from the 1990 National Health Interview SurveyHealth Promotion and Disease Prevention Supplement. Diabetes Care. 1990;1995(18):27-33.

32. Kwon HR, Min KW, Ahn HJ, et al. Effects of aerobic exercise on abdominal fat, thigh muscle mass and muscle strength in type 2 diabetic subject. Korean Diabetes J. 2010;34(1):23-31. 